\title{
A sieve approach to the Waring-Goldbach problem, II On the seven cubes theorem
}

\author{
by \\ JÖRG BRÜDERN (Stuttgart)
}

1. Introduction. It is conjectured that all sufficiently large natural numbers satisfying some necessary congruence condition, are the sum of four cubes of primes. The best result in this direction is due to Hua and dates back to 1938: all large numbers in some congruence class are the sum of nine cubes of primes (see [8]). In the present paper we show that if instead of primes one asks for almost primes of some fixed order $r$ (that is, numbers with at most $r$ prime factors, counted with multiplicity, or $P_{r}$-numbers for short), then seven variables suffice.

TheOREM. Let $v(n)$ be the number of representations of $n$ in the form

$$
n=x^{3}+y_{1}^{3}+y_{2}^{3}+y_{3}^{3}+y_{4}^{3}+y_{5}^{3}+p^{3},
$$

where $p$ denotes a prime, the $y_{i}$ are $P_{5}$-numbers, and $x$ is a $P_{69}$. Then $v(n) \gg n^{4 / 3}(\log n)^{-27}$.

It may be worth pointing out that seven variables are also required for solving the ordinary Waring problem at present (Watson [14], Vaughan $[11,12])$, and that the lower bound for $v(n)$ is essentially of the expected order of magnitude.

Our theorem supplements a similar result on sums of four cubes obtained in part I of this series [4], to which the reader is referred for a more detailed introduction to the subject. The principal idea in part I was a combined application of the circle method and a sieve, and this will be basic here as well. Very roughly speaking, we shall count the solutions of (1.1) with the $y_{i}$ taken from a certain set of numbers with exactly five prime factors. The Hardy-Littlewood method is used to obtain asymptotic formulae for the

This paper is a variation on the theme of Chapter 3 of the author's Habilitationsschrift at Göttingen University [2]. The present version was prepared while the author was enjoying a stay at Mathematisches Forschungsinstitut Oberwolfach, supported by "Förderpreis Algebra-Zahlentheorie 1993". 
number of such solutions subject to an additional constraint $x \equiv 0(\bmod d)$. This allows the linear sieve to be applied to the variable $x$. To press the method home we require, in addition to the tools developed in part I, a mean value estimate for cubic exponential sums from our recent paper [3], and a special device for improving the usual minor arc technology due to Vaughan [11, 12].

We have organized the material in such a way that some fairly technical estimates are provided in the next section, before embarking on the main argument. We shall also have opportunity to fix various notations. In Section 3 we apply the circle method to supply the relevant sieve input. The circle method work is completed in Section 4 by examining the minor arcs. The success of the sieve is then dependent on an upper bound for the solutions of (1.1) where $x$ has large square factors, and in Section 5 a suitable estimate is established to finish the proof.

The methods of this paper extend to Waring's problem with exponents exceeding 3. However, we shall find it more appropriate to comment on this matter and other applications of our technique at the end of the paper.

2. Preliminaries. In this paper $p$ always denotes a prime number, and $\pi$ is reserved for primes $\equiv 2(\bmod 3)$. The same convention applies when subscripts are present. Formulae involving $\varepsilon$ are valid for all $\varepsilon>0$. Our notations are otherwise standard and must be understood from the context, or are explained at the appropriate stage of the argument.

Let

$$
\begin{gathered}
\Theta_{4}=\frac{233}{1815}, \quad \zeta_{1}=\frac{15}{113}\left(1-\Theta_{4}\right), \quad \zeta_{2}=\frac{14}{113}\left(1-\Theta_{4}\right), \quad \zeta_{3}=\frac{84}{113}\left(1-\Theta_{4}\right) ; \\
\Theta_{5}=\frac{3679}{29089}, \quad \theta_{i}=\left(1-\Theta_{5}\right) \zeta_{i}(1 \leq i \leq 3), \quad \theta_{4}=\left(1-\Theta_{5}\right) \Theta_{4} .
\end{gathered}
$$

Of importance later are the sizes of $\Theta_{4}, \Theta_{5}$ and the relations

$$
\Theta_{4}+\zeta_{1}+\zeta_{2}+\zeta_{3}=\Theta_{5}+\theta_{1}+\theta_{2}+\theta_{3}+\theta_{4}=1 \text {. }
$$

Let $\mathcal{A}_{4}(P)$ denote the set of all $n$ which can be written in the form $n=$ $p_{0} p_{1} p_{2} p_{3}$ with $P^{\Theta_{4}}<p_{0} \leq 2 P^{\Theta_{4}}$ and $P^{\zeta_{i}}<p_{i} \leq 2 P^{\zeta_{i}}(i=1,2,3)$. Such a representation is necessarily unique. Similarly, let $\mathcal{A}_{5}(P)$ be the set of all $n$ of the form $n=\pi p_{1} p_{2} p_{3} p_{4}$ with $P^{\Theta_{5}}<\pi \leq 2 P^{\Theta_{5}}$ and $P^{\theta_{i}}<p_{i} \leq 2 P^{\theta_{i}} \quad(i=$ $1,2,3,4)$. Again, such representations of $n$ are unique.

Lemma 1. Let $k=4$ or 5 . Let $S_{k}$ denote the number of solutions of

$$
x_{1}^{3}+y_{1}^{3}+y_{2}^{3}=x_{2}^{3}+y_{3}^{3}+y_{4}^{3}
$$

subject to $x_{i} \leq P$ and $y_{i} \in \mathcal{A}_{k}(P)$. Then

$$
S_{k} \ll P^{3+2 \Theta_{k}+\varepsilon} \text {. }
$$


Proof. These are the special cases $l=4$ and 5 of the Proposition in Brüdern [3].

Lemma 2. Let

$$
M=P^{\Theta_{5}}, \quad Q=P M^{-1} .
$$

Let $T$ denote the number of solutions of

$$
\pi_{1}^{3}\left(y_{1}^{3}-z_{1}^{3}\right)+\pi_{2}^{3}\left(y_{2}^{3}-z_{2}^{3}\right)+\pi_{3}^{3}\left(y_{3}^{3}-z_{3}^{3}\right)=0
$$

subject to $M<\pi_{i} \leq 2 M$ and $y_{i}, z_{i} \in \mathcal{A}_{4}(Q)$. Then $T \ll P^{3}$.

Pr o of. This follows from Lemma 1 by a word for word adoption of the proof of Lemma 3 of Vaughan [12]. We may leave the details to the reader.

Our next lemma concerns the exponential sum

$$
S(q, a)=\sum_{x=1}^{q} e\left(\frac{a x^{3}}{q}\right) .
$$

It also features the multiplicative function $\kappa(q)$ defined on prime powers by

$$
\kappa\left(p^{3 l}\right)=p^{-l}, \quad \kappa\left(p^{3 l+1}\right)=p^{-l-1 / 2}, \quad \kappa\left(p^{3 l+2}\right)=p^{-l-1} \quad(l \geq 0) .
$$

Lemma 3. Let $(a, q)=1$. Then

$$
\sum_{d \leq D} \mu(d)^{2} \frac{\left|S\left(q, a d^{3}\right)\right|}{q d} \ll 8^{\nu(q)} \kappa(q) \log D,
$$

where $\nu(q)$ is the number of different prime factors of $q$.

Proof. By standard estimates for the sum (2.2) such as Lemmata 4.3, 4.4 and 4.5 of Vaughan [9] we have

$$
r^{-1}|S(r, b)| \ll 2^{\nu(r)} \kappa(r)
$$

whenever $(b, r)=1$. Hence the left hand side of the proposed inequality does not exceed

$$
\ll 2^{\nu(q)} \sum_{d \leq D} \mu(d)^{2} d^{-1} \kappa\left(q /\left(q, d^{3}\right)\right) .
$$

We decompose $q$ into $q=t^{3} u$ with cube-free $u$, this factorisation is unique. Now any square-free $d$ can be written as $d=d_{t} d_{u}$ where $d_{t}=(t, d)$. Note that $\left(d_{t}, d_{u}\right)=1$ and

$$
\left(q, d^{3}\right)=\left(t^{3} u, d_{t}^{3} d_{u}^{3}\right)=d_{t}^{3}\left(u, d_{u}^{3}\right)=d_{t}^{3}\left(u, d_{u}^{2}\right)
$$

since $u$ is cube-free and $d$ is square-free. Hence

$$
\kappa\left(\frac{q}{\left(q, d^{3}\right)}\right)=\kappa\left(\frac{t^{3} u}{d_{t}^{3}\left(d_{u}^{2}, u\right)}\right)=t^{-1} d_{t}\left(\frac{u}{\left(d_{u}^{2}, u\right)}\right)^{-1 / 2}=\kappa(q) d_{t}\left(u, d_{u}^{2}\right)^{1 / 2}
$$


since $\kappa(q)=t^{-1} u^{-1 / 2}$. It follows that

$$
\begin{aligned}
\sum_{d \leq D} \mu(d)^{2} d^{-1} \kappa\left(\frac{q}{\left(q, d^{3}\right)}\right) & \leq \kappa(q) \sum_{d_{t} \mid t} \sum_{d_{u} \leq D / d_{t}} \mu\left(d_{t} d_{u}\right)^{2} d_{u}^{-1}\left(u, d_{u}\right) \\
& \leq \kappa(q) \sum_{d_{t} \mid t} \sum_{\delta \mid u} \sum_{l \leq D} l^{-1} \mu\left(d_{t} \delta\right)^{2} \ll \kappa(q) 2^{2 \nu(q)} \log D
\end{aligned}
$$

as required.

Lemma 4. Let $\mathfrak{N}(q, a)$ denote the interval $|q \alpha-a| \leq P^{-2}$, and write $\mathfrak{N}$ for the union of all $\mathfrak{N}(q, a)$ with $1 \leq a \leq q \leq P$ and $(a, q)=1$. Define $a$ function $G$ on $\mathfrak{N}$ by

$$
G(\alpha)=8^{\nu(q)} \kappa(q)\left(1+P^{3}\left|\alpha-\frac{a}{q}\right|\right)^{-1}
$$

when $\alpha \in \mathfrak{N}(q, a)$. Then

$$
\int_{\mathfrak{N}} G(\alpha)^{2}\left|\sum_{P<p \leq 2 P} e\left(\alpha p^{3}\right)\right|^{2} d \alpha \ll P^{-1}(\log P)^{192} .
$$

Proof. By the orthogonality of additive characters,

$$
\begin{aligned}
& \int_{\mathfrak{N}} G(\alpha)^{2}\left|\sum_{P<p \leq 2 P} e\left(\alpha p^{3}\right)\right|^{2} d \alpha \\
& \ll \sum_{q \leq P} 64^{\nu(q)} \kappa(q)^{2} \sum_{a=1}^{q} \int_{-\infty}^{\infty}\left|\sum_{P<p \leq 2 P} e\left(\left(\frac{a}{q}+\beta\right) p^{3}\right)\right|^{2}\left(1+P^{3}|\beta|\right)^{-2} d \beta \\
& \ll P^{-3} \sum_{q \leq P} 64^{\nu(q)} \kappa(q)^{2} q \psi(q),
\end{aligned}
$$

where $\psi(q)$ is the number of solutions of the congruence $p_{1}^{3} \equiv p_{2}^{3}(\bmod q)$ with $P<p_{1}, p_{2} \leq 2 P$. For $q \leq P$ we must have $\left(p_{1} p_{2}, q\right)=1$. For $(a, q)=1$ the number of solutions of the congruence $x^{3} \equiv a(\bmod q)$ with $1 \leq x \leq q$, $(x, q)=1$ does not exceed $O\left(3^{\nu(q)}\right)$. Hence, once $p_{2}$ is fixed, the number of choices for $p_{1}$ is $\ll 3^{\nu(q)} P q^{-1}$. Hence, for $q \leq P$ we have $\psi(q) \ll 3^{\nu(q)} q^{-1} P^{2}$. We deduce that the integral in question is bounded by

$$
\ll P^{-1} \sum_{q \leq P} 192^{\nu(q)} \kappa(q)^{2} \ll P^{-1} \prod_{p \leq P}\left(1+\frac{192}{p}+O\left(p^{-2}\right)\right),
$$

and the lemma follows.

3. The circle method and the linear sieve. We now prepare the ground for the application of a sieve which will ultimately yield the Theorem. Our application of the Hardy-Littlewood method involves the exponential 
sums

$f_{d}(\alpha)=\sum_{P / d<y \leq 2 P / d} e\left(\alpha d^{3} y^{3}\right), \quad g(\alpha)=\sum_{P<p \leq 2 P} e\left(\alpha p^{3}\right), \quad h(\alpha)=\sum_{y \in \mathcal{A}_{5}(P)} e\left(\alpha y^{3}\right)$, where $P=\frac{1}{10} n^{1 / 3}$. For a measurable set $\mathfrak{B}$ we define

$$
v_{d}(n, \mathfrak{B})=\int_{\mathfrak{B}} f_{d}(\alpha) g(\alpha) h(\alpha)^{5} e(-\alpha n) d \alpha .
$$

The significance for our problem emerges from the fact that $v_{d}(n,[0,1])=$ $v_{d}(n)$, say, counts the solutions $(1.1)$ with $x \equiv 0(\bmod d), P<x, p \leq 2 P$ and $y_{i} \in \mathcal{A}_{5}(P)$.

The goal is to find asymptotic formulae of the shape $v_{d}(n)=\frac{\omega(d)}{d} X+$ $R(d, n)$, where $X$ is some function of $n$, where $\omega(d)$ is multiplicative, and where $R(n, d)$ is small on average over $d$. With this end in view we define major arcs by writing

$$
L=(\log n)^{5000}, \quad \mathfrak{M}(q, a)=\left\{\alpha:\left|\alpha-\frac{a}{q}\right| \leq L P^{-3}\right\}
$$

and then introduce $\mathfrak{M}$ as the union of all $\mathfrak{M}(q, a)$ with $1 \leq a \leq q \leq L$, $(a, q)=1$. As usual, the main term arises from the set $\mathfrak{M}$. This can be seen by straightforward arguments so we shall be brief. Let $\alpha \in \mathfrak{M}(q, a)$ with $q \leq L$. By Theorem 4.1 of Vaughan [9] we have

$$
f_{d}(\alpha)=\frac{S\left(q, a d^{3}\right)}{q} J\left(d^{3}\left(\alpha-\frac{a}{q}\right), \frac{P}{d}\right)+O(L)
$$

uniformly for $d \leq P^{1 / 2}$, say. Here we have written

$$
J(\beta, \xi)=\int_{\xi}^{2 \xi} e\left(\beta t^{3}\right) d t
$$

By Euler's summation formula, we have $J(\beta, P)=u(\beta)+O(1+P|\beta|)$, where

$$
u(\beta)=\frac{1}{3} \sum_{P^{3}<x \leq 8 P^{3}} x^{-2 / 3} e(\beta x),
$$

and a change of variable now shows that

$$
f_{d}(\alpha)=\frac{S\left(q, a d^{3}\right)}{q d} u(\beta)+O(L),
$$

where $\beta=\alpha-a / q$. The approximation to $g(\alpha)$ is similar, but involves the data

$$
S^{*}(q, a)=\sum_{\substack{x=1 \\(x, q)=1}}^{q} e\left(\frac{a x^{3}}{q}\right), \quad w(\beta, \xi)=\frac{1}{3} \sum_{\xi^{3}<x \leq 8 \xi^{3}} e(\beta x) x^{-2 / 3}(\log x)^{-1} .
$$


For $q \leq L,|\beta| \leq L P^{-3}$, Lemma 6 of Hua [7] shows that

$$
g\left(\frac{a}{q}+\beta\right)=\varphi(q)^{-1} S^{*}(q, a) w(\beta, P)+O\left(P(\log P)^{-A}\right)
$$

for any $A>0$. An analogous result for $h(\alpha)$ requires a little more care. We write $\mathcal{B}$ to denote the set of all numbers $b=\pi p_{1} p_{2} p_{3}$ where $P^{\Theta_{5}}<\pi \leq 2 P^{\Theta_{5}}$ and $P^{\theta_{i}}<p_{i} \leq 2 P^{\theta_{i}}(i=1,2,3)$. Still assuming $\alpha=a / q+\beta \in \mathfrak{M}(q, a)$ we can now write

$$
\begin{aligned}
h(\alpha) & =\sum_{b \in \mathcal{B}} \sum_{P^{\theta_{4}}<p \leq 2 P^{\theta_{4}}} e\left(\alpha b^{3} p^{3}\right) \\
& =\sum_{b \in \mathcal{B}}\left(\frac{S^{*}\left(q, a b^{3}\right)}{\varphi(q)} w\left(\beta b^{3}, P^{\theta_{4}}\right)+O_{A}\left(\frac{P^{\theta_{4}}}{(\log P)^{A}}\right)\right)
\end{aligned}
$$

after applying Hua's result again to the inner sum. When $q \leq L$ and $n$ is sufficiently large, we have $(b, q)=1$ for any $b \in \mathcal{B}$, which implies $S^{*}\left(q, a b^{3}\right)=$ $S^{*}(q, a)$. It now follows that

$$
h(\alpha)=\frac{S^{*}(q, a)}{\varphi(q)} W(\beta)+O_{A}\left(P(\log P)^{-A}\right),
$$

where

$$
W(\beta)=\sum_{b \in \mathcal{B}} w\left(\beta b^{3}, P^{\theta_{4}}\right) .
$$

By integrating over $\mathfrak{M}$ we deduce from (3.2), (3.3) and (3.4) that

$$
\begin{aligned}
v_{d}(n, \mathfrak{M})= & \sum_{q \leq L} \sum_{\substack{a=1 \\
(a, q)=1}}^{q} \frac{S\left(q, a d^{3}\right) S^{*}(q, a)^{6}}{d q \varphi(q)^{6}} e\left(-\frac{a n}{q}\right) I(n) \\
& +O\left(P^{4} d^{-1}(\log P)^{-A}\right)
\end{aligned}
$$

where

$$
I(n)=\int_{-L P^{-3}}^{L P^{-3}} u(\beta) w(\beta, P) W(\beta)^{5} e(-\beta n) d \beta,
$$

which still holds uniformly for $d \leq P^{1 / 2}$. The singular integral (3.7) can be evaluated by a routine argument. One has $u(\beta) \ll|\beta|^{-1}$ for $P^{-3}<|\beta| \leq 1 / 2$ and a similar bound for $w(\beta, P)$, by partial summation. Hence the range of integration in (3.7) can be extended to $\left[-\frac{1}{2}, \frac{1}{2}\right]$, at the cost of an error $O\left(P^{4} L^{-1 / 2}\right)$; and the integration over $\left[-\frac{1}{2}, \frac{1}{2}\right]$ equals a weighted counting of the solution of a linear equation. This yields the inequalities

$$
n^{4 / 3}(\log n)^{-26} \ll I(n) \ll n^{4 / 3}(\log n)^{-26} .
$$


Now we complete the singular series in (3.6). By Lemma 5 of Hua [7], one has $\left|S^{*}(q, a)\right| \ll q^{1 / 2+\varepsilon}$ for $(a, q)=1$. Hence, the series

$$
\mathfrak{S}_{d}(n)=\sum_{q=1}^{\infty} \sum_{\substack{a=1 \\(a, q)=1}}^{q} \frac{S\left(q, a d^{3}\right) S^{*}(q, a)^{6}}{q \varphi(q)^{6}} e\left(-\frac{a n}{q}\right)
$$

converges absolutely, and is bounded by $O(1)$, uniformly in $n$ and $d$; and from (3.6) and (3.8) we see that

$$
v_{d}(n, \mathfrak{M})=\mathfrak{S}_{d}(n) I(n) d^{-1}+O\left(P^{4} d^{-1}(\log P)^{-A}\right) .
$$

By routine arguments it is readily seen that $\mathfrak{S}_{1}(n) \gg 1$ for all $n$. For square-free $d$ we can now define

$$
\omega(d)=\mathfrak{S}_{d}(n) \mathfrak{S}_{1}(n)^{-1} .
$$

By a trivial modification of the arguments in Section 4 of part I [4] (where the analogous function for four cubes is considered) it is readily shown that $\omega(d)$ is multiplicative, and that

$$
\frac{\omega(p)}{p}=\frac{M(p, n)}{M^{*}(p, n)}(p \neq 3), \quad \frac{\omega(3)}{3}=\frac{M(9, n)}{M^{*}(9, n)},
$$

where $M(q, n)$ equals the number of incongruent solutions of

$$
y_{1}^{3}+\ldots+y_{6}^{3} \equiv n(\bmod q), \quad\left(q, y_{1} \ldots y_{6}\right)=1,
$$

and $M^{*}(q, n)$ equals the number of incongruent solutions of

$$
x^{3}+y_{1}^{3}+\ldots+y_{6}^{3} \equiv n(\bmod q), \quad\left(q, y_{1} \ldots y_{6}\right)=1 .
$$

Still following the line of investigation of part I, Section 4, we now find that

$$
\omega(p)=1+O\left(p^{-1 / 2}\right),
$$

where the implicit constant is independent of $n$. It is clear that $\omega(p) \geq$ 0 , but for the sieve we also require $\omega(p)<p$, and this entails a slight complication. In fact, for $p \neq 3$, the condition $\omega(p)<p$ is equivalent to $M(p, n)<M^{*}(p, n)$, and this is the case if and only if the defining congruence for $M^{*}(p, n)$ has a solution with $p \nmid x$. By the methods of [4] (or Chapter 2 of Vaughan [9]) this is readily confirmed for $p \geq 5$. The primes $p=2$ and 3 are exceptional here in the sense that the truth of the inequalities $\omega(2)<2, \omega(3)<3$ depends on congruence conditions on $n$ ( $\bmod 18)$. Indeed, by a direct counting, it is seen that $\omega(2)=2$ for $n \equiv 0$ $(\bmod 2)$, and $\omega(3)=3$ for $n \equiv 0(\bmod 9)$, but that in all other cases one has $\omega(2)<2, \omega(3)<3$. If $n$ is neither divisible by 2 nor 9 , we are ready to apply the linear sieve theorem of Greaves [6]. If $v^{*}(n)$ denotes the number 
of solutions of (1.1) with $y_{i} \in \mathcal{A}_{5}(P)$ and $P_{67}$-numbers $x$, then

$v^{*}(n) \gg X \prod_{p \leq D}\left(1-\frac{\omega(p)}{p}\right)^{-1}-\left|\sum_{d \leq D} \eta_{d}\left(v_{d}(n)-\frac{\omega(d)}{d} X\right)\right|-\sum_{p>P^{\delta}} v_{p^{2}}(n)$, where $X=\mathfrak{S}_{1}(n) I(n)$, where $\eta_{d}$ are certain complex numbers satisfying $\left|\eta_{d}\right| \leq 1$ and $\eta_{d}=0$ for non-square-free $d$, where $\delta$ is some positive real number (which we may suppose to be as small as we like), and where

$$
D=P^{1 / 66} \text {. }
$$

From (3.9) we have

$$
v_{d}(n)-\frac{\omega(d)}{d} X=v_{d}(n, \mathfrak{m})+O\left(d^{-1} P^{4}(\log P)^{-A}\right),
$$

where $\mathfrak{m}=\left[L P^{-3}, 1+L P^{-3}\right] \backslash \mathfrak{M}$ are the minor arcs. Therefore it now suffices to establish the estimates

$$
\begin{gathered}
\sum_{d \leq D} \eta_{d} v_{d}(n, \mathfrak{m}) \ll P^{4}(\log P)^{-50}, \\
\sum_{p>P^{\delta}} v_{p^{2}}(n) \ll P^{4-\delta / 5},
\end{gathered}
$$

to deduce from (3.10) and (3.8) that $v^{*}(n) \gg n^{4 / 3}(\log n)^{-27}$. This gives the Theorem for odd $n \not \equiv 0(\bmod 9)$ with the slightly superior outcome of finding a $P_{67}$ for $x$.

Only simple modifications in the above argument are required to cover even $n$ and $n \equiv 0(\bmod 9)$. Instead of $(1.1)$ we consider the equation

$$
2^{\gamma(2)} 3^{\gamma(3)} z^{3}+p^{3}+y_{1}^{3}+\ldots+y_{5}^{3}=n,
$$

where $\gamma(2)=3$ if $n$ is even, and $\gamma(2)=0$ if $n$ is odd, and where $\gamma(3)=3$ or 0 according to $n \equiv 0(\bmod 9)$ or not. By the same method, we find solutions with $y_{i} \in \mathcal{A}_{5}(P)$ and $P_{67}$-numbers $z$, and the Theorem follows in the exceptional cases also.

It remains to verify (3.12) and (3.13). This is the theme of the next two sections.

4. The minor arcs. In this section we prove (3.12). We write

$$
F(\alpha)=\sum_{d \leq D} \eta_{d} f_{d}(\alpha)
$$

and then deduce from (3.1) that

$$
\left|\sum_{d \leq D} \eta_{d} v_{d}(n, \mathfrak{m})\right| \leq \int_{\mathfrak{m}}\left|F(\alpha) g(\alpha) h(\alpha)^{5}\right| d \alpha .
$$


The minor arcs are split into two subsets which are treated by different methods. We recall the abbreviation $M=P^{\Theta_{5}}$ from (2.1) and then put $R=P M^{3} D^{-1}$. Let $\mathfrak{K}$ denote the union of all intervals

$$
\mathfrak{K}(q, a)=\left\{\alpha:|q \alpha-a| \leq R P^{-3}\right\}
$$

with $1 \leq a \leq q \leq R$ and $(a, q)=1$. Let $\mathfrak{k}$ denote the complement of $\mathfrak{K}$ in $[0,1]$ modulo 1 . Our first task is to estimate the contribution to (4.2) arising from $\mathfrak{k}$. We shall mainly be concerned with proving the estimate

$$
\int_{\mathfrak{k}}\left|F(\alpha) h(\alpha)^{3}\right|^{2} d \alpha \ll P^{5-\delta} M^{-2}
$$

for some $\delta>0$. If this is taken for granted, we only need to add to this the bound

$$
\int_{0}^{1}\left|g(\alpha) h(\alpha)^{2}\right|^{2} d \alpha \ll P^{3+\varepsilon} M^{2},
$$

which follows from Lemma 1 on considering the underlying diophantine equation; Schwarz's inequality then yields

$$
\int_{\mathfrak{k}}\left|F(\alpha) g(\alpha) h(\alpha)^{5}\right| d \alpha \ll P^{4-\delta / 2+\varepsilon}
$$

as required.

Our proof of (4.3) follows Vaughan [12] quite closely so we may be brief. We write

$$
f(\alpha, k, Y)=\sum_{\substack{Y<y \leq 2 Y \\(y, k)=1}} e\left(\alpha y^{3}\right)
$$

and then have

$$
f_{d}(\alpha)=f\left(\alpha d^{3}, 1, P / d\right)=f\left(\alpha d^{3}, p, P / d\right)+f\left(\alpha(p d)^{3}, 1, P /(p d)\right)
$$

for any prime $p$. Accordingly we decompose (4.1) as

$$
F(\alpha)=F_{1}(\alpha, p)+F_{2}(\alpha, p),
$$

where

$$
F_{1}(\alpha, p)=\sum_{d \leq D} \eta_{d} f\left(\alpha d^{3}, p, P / d\right), \quad F_{2}(\alpha, p)=\sum_{d \leq D} \eta_{d} f\left(\alpha(p d)^{3}, 1, P /(p d)\right) .
$$

The definition of $\mathcal{A}_{5}(P)$ enables us to write

$$
h(\alpha)=\sum_{M<\pi \leq 2 M} H\left(\alpha \pi^{3}\right),
$$

where

$$
H(\alpha)=\sum_{y \in \mathcal{A}_{4}(P / M)} e\left(\alpha y^{3}\right) .
$$


We can now conclude that

$$
\left|F(\alpha) h(\alpha)^{3}\right| \leq\left(\sum_{M<\pi \leq 2 M}|F(\alpha)|^{1 / 3}\left|H\left(\alpha \pi^{3}\right)\right|\right)^{3} \ll U_{1}(\alpha)+U_{2}(\alpha),
$$

where

$$
U_{j}(\alpha)=\left(\sum_{M<\pi \leq 2 M}\left|F_{j}(\alpha, \pi)\right|^{1 / 3}\left|H\left(\alpha \pi^{3}\right)\right|\right)^{3} .
$$

We first concentrate on $U_{2}$. If $\alpha \in \mathfrak{k}$ and $d \leq D, M<p \leq 2 M$ then $f\left(\alpha d^{3} p^{3}, 1, P /(d p)\right) \ll\left(P D^{-1} M^{-1}\right)^{3 / 4+\varepsilon}$ by a routine application of Weyl's inequality (see Vaughan [12], p. 213 for more details). Hence

$$
U_{2}(\alpha) \ll P^{3 / 4+\varepsilon} D^{1 / 4} M^{-3 / 4}\left(\sum_{M<\pi \leq 2 M}\left|H\left(\alpha \pi^{3}\right)\right|\right)^{3}
$$

for $\alpha \in \mathfrak{k}$. By considering the underlying diophantine equation, we deduce from Lemma 2 that

$$
\int_{0}^{1}\left(\sum_{M<\pi \leq 2 M}\left|H\left(\alpha \pi^{3}\right)\right|^{2}\right)^{3} d \alpha \ll P^{3+\varepsilon}
$$

and then infer from Cauchy's inequality

$$
\begin{aligned}
\int_{\mathfrak{k}} U_{2}(\alpha)^{2} d \alpha & \ll P^{3 / 2+\varepsilon} D^{1 / 2} M^{3 / 2} \int_{0}^{1}\left(\sum_{M<\pi \leq 2 M}\left|H\left(\alpha \pi^{3}\right)\right|^{2}\right)^{3} d \alpha \\
& \ll P^{9 / 2+2 \varepsilon} D^{1 / 2} M^{3 / 2} \ll P^{5-\delta} M^{-2}
\end{aligned}
$$

for some $\delta>0$, as is readily seen from (3.11).

The treatment of $U_{1}(\alpha)$ is less straightforward but we can heavily borrow from Vaughan [12]. We begin by applying Hölder's inequality to the defining equation for $U_{1}$. This gives

$$
\begin{array}{rl}
\int_{\mathfrak{k}} U_{1}(\alpha)^{2} & d \alpha \\
& \leq D M^{5} \sum_{d \leq D} \sum_{M<\pi \leq 2 M} \int_{\mathfrak{k}}\left|f\left(\alpha d^{3}, \pi, P / d\right)\right|^{2}\left|H\left(\alpha \pi^{3}\right)\right|^{6} d \alpha .
\end{array}
$$

Let $\mathfrak{w}$ denote the set of all $\alpha \in[0,1]$ such that $|q \alpha-a| \leq R P^{-3},(a, q)=$ 1 implies that $q>R M^{-3}=P D^{-1}$. By an obvious adjustment of the argument on p. 214 of Vaughan [12] we find that

$$
\int_{\mathfrak{k}}\left|f\left(\alpha d^{3}, \pi, P / d\right)\right|^{2}\left|H\left(\alpha \pi^{3}\right)\right|^{6} d \alpha \leq \int_{\mathfrak{w}}\left(P d^{-1}+2 \operatorname{Re} \Phi_{\pi, d}(\alpha)\right)|H(\alpha)|^{6} d \alpha,
$$


where

$$
\Phi_{\pi, d}(\alpha)=\sum_{l \leq 8 P d^{-1} \pi^{-3}} \sum_{\substack{2 P d^{-1}+l \pi^{3}<z \leq 4 P d^{-1}-l \pi^{3} \\ z \equiv l(\bmod 2)}} e\left(\alpha l d^{3}\left(\frac{3}{4} z^{2}+\frac{1}{4} l^{2} \pi^{6}\right)\right) .
$$

As we shall see in a moment, one has

$$
\sup _{\substack{\alpha \in \mathfrak{w} \\ M<\pi \leq 2 M}} \sum_{d \leq D}\left|\Phi_{\pi, d}(\alpha)\right| \ll P^{3 / 2+\varepsilon} M^{-3} D^{1 / 2} .
$$

The case $k=4$ of Lemma 1 shows

$$
\int_{0}^{1}|H(\alpha)|^{6} d \alpha \ll(P / M)^{3+2 \Theta_{4}+\varepsilon}
$$

so that from (4.8) we can now deduce that

$$
\begin{aligned}
\int_{\mathfrak{k}} U_{1}(\alpha)^{2} d \alpha & \ll D M^{6}\left(P^{1+\varepsilon}+P^{3 / 2+\varepsilon} M^{-3} D^{1 / 2}\right)(P / M)^{3+2 \Theta_{4}+\varepsilon} \\
& \ll P^{5-\delta} M^{-2}
\end{aligned}
$$

providing we can show that

$$
D^{3 / 2}<P^{1 / 2-2\left(\Theta_{4}+\Theta_{5}-\Theta_{4} \Theta_{5}+\delta\right)} .
$$

A simple numerical check confirms this if $\delta>0$ is sufficiently small (use (3.11)). By (4.6), (4.7) and (4.10) we see that (4.3) holds, but it remains to verify (4.9). This is a standard exercise but we give an outline for completeness. By Weyl's differencing technique one has

$$
\begin{aligned}
\left|\sum_{d \leq D} \Phi_{\pi, d}(\alpha)\right|^{2} & \ll P^{1+\varepsilon} \pi^{-3} \sum_{d \leq D} \sum_{l \leq 8 P d^{-1} \pi^{-3}}\left|\sum_{z} e\left(\frac{3}{4} \alpha d^{3} l z^{2}\right)\right|^{2} \\
\ll & P^{3+\varepsilon} \pi^{-6} \\
& +P^{1+\varepsilon} \pi^{-3} \sum_{d \leq D} \sum_{l \leq 8 P d^{-1} \pi^{-3}} \sum_{j \ll P / d} \min \left(P / d,\left\|\alpha d^{3} l j\right\|^{-1}\right) .
\end{aligned}
$$

We split the range for $d$ into $O(\log D)$ parts of the shape $\Delta<d \leq 2 \Delta$. By a standard divisor argument we deduce that the previous expression does not exceed

$$
\ll P^{3+\varepsilon} \pi^{-6}+P^{1+2 \varepsilon} \pi^{-3} \sum_{u \ll P^{2} \Delta \pi^{-3}} \min \left(P \Delta^{-1},\|\alpha u\|^{-1}\right)
$$

for some $\Delta$ with $1 \leq \Delta \leq D$. For $|q \alpha-a| \leq q^{-1}$ and $(q, a)=1$ Lemma 2.2 of Vaughan [9] shows that

$$
\left|\sum_{d \leq D} \Phi_{\pi, d}(\alpha)\right|^{2} \ll P^{\varepsilon}\left(\frac{P^{3} \Delta}{\pi^{6}}+\frac{P^{4}}{q \pi^{6}}+\frac{q P}{\pi^{3}}\right) .
$$


If $M<\pi \leq 2 M$ this gives (4.9) when $P D^{-1} \ll q \ll P^{2} M^{-3} D$. By Dirichlet's theorem on diophantine approximation it is readily seen that $q$ can be chosen in this range for all $\alpha \in \mathfrak{w}$, and (4.9) follows. The proof of (4.3) is now complete.

It now remains to consider the set $\mathfrak{K} \cap \mathfrak{m}$ which is treated more like major arcs. We begin by refining the argument used to verify (3.2). In the notation used there, we deduce from Theorem 2 of Vaughan [10] that

$$
f_{d}\left(\frac{a}{q}+\beta\right)=\frac{S\left(q, a d^{3}\right)}{q d} J(\beta)+O\left(q^{\varepsilon}\left(q+P^{3}|q \alpha-a|\right)^{1 / 2}\right) .
$$

Now let $\alpha \in \mathfrak{K}(q, a)$. Let $G$ be the function defined in Lemma 4 (extended in the natural way to $\mathfrak{K}$ ). By summing the previous equation over $d$, we infer from (4.1), Lemma 3 and a standard bound for $J(\beta)$ that

$$
F(\alpha) \ll P G(\alpha)+D R^{1 / 2+\varepsilon} \quad(\alpha \in \mathfrak{K}) .
$$

By Lemma 1, and considering the underlying diophantine equation,

$$
\int_{0}^{1}|h(\alpha)|^{6} d \alpha \ll P^{3+\varepsilon} M^{2},
$$

and by Schwarz's inequality, (4.4) and (4.11),

$$
\int_{0}^{1}\left|g(\alpha) h(\alpha)^{5}\right| d \alpha \ll P^{3+\varepsilon} M^{2} .
$$

It now follows that

$$
\int_{\mathfrak{K} \cap \mathfrak{m}}\left|F(\alpha) g(\alpha) h(\alpha)^{5}\right| d \alpha \ll P \int_{\mathfrak{K} \cap \mathfrak{m}} G(\alpha)\left|g(\alpha) h(\alpha)^{5}\right| d \alpha+D R^{1 / 2} P^{3+2 \varepsilon} M^{2} .
$$

The second term on the right is $O\left(P^{4-\delta}\right)$ for some $\delta>0$, as is readily checked. Moreover, if $\mathfrak{N}$ is the set introduced in Lemma 4, we deduce from the trivial bound $\kappa(q) \leq q^{-1 / 3}$ that

$$
\sup _{\alpha \in \mathfrak{K} \backslash \mathfrak{N}} G(\alpha) \ll P^{\varepsilon-1 / 3}
$$

Using (4.12) once again, we see that

$$
\int_{\mathfrak{k} \cap \mathfrak{m}}\left|F(\alpha) g(\alpha) h(\alpha)^{5}\right| d \alpha \ll P \int_{\mathfrak{N} \cap \mathfrak{m}} G(\alpha)\left|g(\alpha) h(\alpha)^{5}\right| d \alpha+P^{4-\delta} ;
$$

and the proof of (3.12) is now completed by verifying the bound

$$
\int_{\mathfrak{N} \cap \mathfrak{m}} G(\alpha)\left|g(\alpha) h(\alpha)^{5}\right| d \alpha \ll P^{3}(\log P)^{-50} .
$$


To see this we observe that by Theorem 2 of Vaughan [11] and considering the underlying diophantine equations one has

$$
\int_{0}^{1}|g(\alpha)|^{8} d \alpha \ll P^{5}, \quad \int_{0}^{1}|h(\alpha)|^{8} d \alpha \ll P^{5} .
$$

Moreover, the definitions of $\mathfrak{m}$ and $G$ readily show that

$$
\sup _{\alpha \in \mathfrak{N} \cap \mathfrak{m}} G(\alpha) \ll L^{\varepsilon-1 / 3} \text {. }
$$

Now Hölder's inequality yields

$$
\int_{\mathfrak{N} \cap \mathfrak{m}} G\left|g h^{5}\right| d \alpha \ll L^{-1 / 10}\left(\int_{\mathfrak{N}} G^{2}|g|^{2} d \alpha\right)^{1 / 3}\left(\int_{0}^{1}|g|^{8} d \alpha\right)^{1 / 24}\left(\int_{0}^{1}|h|^{8} d \alpha\right)^{5 / 8} .
$$

The first integral is estimated in Lemma 4, the other two in (4.14). This establishes (4.13) and (3.12).

5. Large square factors. Finally, we establish (3.13). The point of departure is the obvious inequality

$$
\sum_{p>P^{\delta}} v_{p^{2}}(n) \leq V(n)
$$

where $V(n)$ denotes the number of solutions of

$$
p^{6} z^{3}+x^{3}+y_{1}^{3}+y_{2}^{3}+y_{3}^{3}+y_{4}^{3}+y_{5}^{3}=n
$$

with

$$
p>P^{\delta}, \quad P<p^{2} z \leq 2 P, \quad P<x \leq 2 P, \quad y_{i} \in \mathcal{A}_{5}(P) .
$$

Using the notation from the previous sections we can write $V(n)$ as an integral,

$$
V(n)=\sum_{p>P^{\delta}} \int_{0}^{1} f_{p^{2}}(\alpha) f_{1}(\alpha) h(\alpha)^{5} e(-\alpha n) d \alpha
$$

and then estimate this integral by the Hardy-Littlewood method, in much the same way as in Sections 3-4. Since we are only interested in an upper bound, the details are simpler. We write

$$
\Xi(\alpha)=\sum_{p>P^{\delta}} f_{p^{2}}(\alpha)
$$

in the interest of brevity. Further progress is now dependent on the estimates

$$
\int_{0}^{1}\left|\Xi(\alpha) h(\alpha)^{2}\right|^{2} d \alpha \ll P^{3+\varepsilon} M^{2}
$$




$$
\int_{0}^{1}|\Xi(\alpha) h(\alpha)|^{4} d \alpha \ll P^{5-\delta} .
$$

To verify (5.5) we observe that the integral equals the number of solutions of

$$
p_{1}^{6} z_{1}^{3}+p_{2}^{6} z_{2}^{3}-p_{3}^{6} z_{3}^{3}-p_{4}^{6} z_{4}^{3}=y_{1}^{3}+y_{2}^{3}-y_{3}^{3}-y_{4}^{3}
$$

subject to

$$
p>P^{\delta}, \quad P<p_{i}^{2} z_{i}<2 P, \quad y_{i} \in \mathcal{A}_{5}(P) .
$$

Let $\mathcal{W}$ be the set of all numbers $w$ which can be written as $w=p^{2} z$ with $p>P^{\delta}, P<w \leq 2 P$. Then $\# \mathcal{W} \ll P^{1-\delta}$, and the number of possible representations of $w$ in the form $p^{2} z$ with $p>P^{\delta}$ is $O(1)$. Hence the integral in (5.5) is $O(K)$, where $K$ is the number of solutions to

$$
w_{1}^{3}+w_{2}^{3}-w_{3}^{3}-w_{4}^{3}=y_{1}^{3}+y_{2}^{3}-y_{3}^{3}-y_{4}^{3}
$$

with $w_{i} \in \mathcal{W}$ and $y_{i} \leq 32 P$. By Lemma 3 of Vaughan [11],

$$
K \ll(\# \mathcal{W})^{2} P^{3+\varepsilon} \ll P^{5-\delta} .
$$

This gives (5.5). In exactly the same way, (5.4) follows from Lemma 1.

Now let $\mathfrak{k}, \mathfrak{K}$ be the pair of major and minor arcs introduced in Section 4 . We observe that on choosing $\eta_{1}=1, \eta_{d}=0(d>1)$ the sum $F(\alpha)$ in (4.1) reduces to $f_{1}(\alpha)$. Hence we may quote from (4.3) the bound

$$
\int_{\mathfrak{k}}\left|f_{1}(\alpha)\right|^{2}|h(\alpha)|^{6} d \alpha \ll P^{5-\delta} M^{-2}
$$

providing $\delta$ is sufficiently small, as we may suppose. By Schwarz's inequality and (5.4) it follows that

$$
\int_{\mathfrak{k}}\left|\Xi(\alpha) f_{1}(\alpha) h(\alpha)^{5}\right| d \alpha \ll P^{4-\delta / 3},
$$

which is acceptable.

The treatment of $\mathfrak{K}$ is straightforward. For $\alpha \in \mathfrak{K}(q, a)$ we deduce from Theorem 2 of Vaughan [10] and standard estimates that

$$
\left|f_{1}(\alpha)\right| \ll\left|f^{*}(\alpha)\right|+R^{1 / 2+\varepsilon},
$$

where

$$
f^{*}(\alpha)=q^{-1}|S(q, a)| P\left(1+P^{3}\left|\alpha-\frac{a}{q}\right|\right)^{-1} .
$$

By (5.4), (4.11) and Schwarz's inequality,

$$
\int_{0}^{1}\left|\Xi(\alpha) h(\alpha)^{5}\right| d \alpha \ll P^{3+\varepsilon} M^{2},
$$


and in the previous section we have already seen that $P^{3+2 \varepsilon} M^{2} R^{1 / 2} \ll P^{4-\delta}$ for some $\delta>0$. From (5.7) and (5.8) we now infer that

$$
\int_{\mathfrak{K}}\left|\Xi f_{1} h^{5}\right| d \alpha \ll \int_{\mathfrak{K}}\left|\Xi f^{*} h^{5}\right| d \alpha+P^{4-\delta} .
$$

The bound

$$
\int_{\mathfrak{K}}\left|f^{*}(\alpha)\right|^{4} d \alpha \ll P^{1+\varepsilon}
$$

is readily established by appeal to Lemma 4.9 of Vaughan [9], and by Hölder's inequality, (5.5) and (4.14) it follows that

$$
\begin{aligned}
\int_{\mathfrak{K}}\left|\Xi f^{*} h^{5}\right| d \alpha & \leq\left(\int_{\mathfrak{K}}\left|f^{*}\right|^{4} d \alpha\right)^{1 / 4}\left(\int_{0}^{1}|\Xi h|^{4} d \alpha\right)^{1 / 4}\left(\int_{0}^{1}|h|^{8} d \alpha\right)^{1 / 2} \\
& \leq P^{4-1 / 5-\delta} .
\end{aligned}
$$

By (5.1), (5.2), (5.3), (5.6), (5.9) and (5.10), this gives (3.13).

6. Further applications of the method. The methods of this paper are by no means limited to Waring's problem for cubes. There is actually an underlying principle for solving diophantine equations in almost primes which may be described as follows. Suppose we are interested in representations of $n$ in the form

$$
n=F\left(x_{1}, \ldots, x_{s}, y_{1}, \ldots, y_{t}\right)
$$

where $F$ is a polynomial with integer coefficients. If the circle method succeeds to establish an asymptotic formula for the number of solutions of (6.1) in a large box $\left|x_{i}\right| \leq P,\left|y_{i}\right| \leq P$ with $y_{i}$ certain $P_{r}$-numbers, then it is usually possible to establish such an asymptotic formula also for the solutions subject to the additional constraints $x_{i} \equiv 0\left(\bmod d_{i}\right)(1 \leq i \leq s)$, uniformly at least in a short range $d_{i} \leq D(1 \leq i \leq s)$, say. If $D$ happens to be a positive power of $P$ then a sieve will find solutions of (6.1) with all the variables almost primes of some fixed order.

This principle can be turned into rigorous results at least when $F$ is an additive polynomial, and in particular for Waring's problem. As usual, let $G(k)$ be the minimal $s$ such that for all sufficiently large $n$ the equation

$$
n=x_{1}^{k}+\ldots+x_{s}^{k}
$$

has solutions in positive integers. If the object of the exercise is to solve (6.2) in primes, then $n$ is required to satisfy the congruence condition, that is, the congruence $x_{1}^{k}+\ldots+x_{s}^{k} \equiv n(\bmod q)$ must be soluble with $\left(q, x_{1} \ldots x_{s}\right)=1$, for all natural numbers $q>1$. Let $\mathcal{W}_{k, s}$ be the set of all integers satisfying the congruence conditions. It is not difficult to see that for $s \geq k$ (say), $\mathcal{W}_{k, s}$ contains an arithmetic progression $s \bmod K$ where $K$ depends only on $k$, 
and hence has positive density. Now let $H_{r}(k)$ be the smallest number $s$ such that for sufficiently large $n \in \mathcal{W}_{k, s}$ there are solutions to (6.2) in $P_{r}$-numbers.

The most modern versions of the circle method such as in Vaughan [13] and Wooley [15] provide asymptotic formulae for the number of solutions of (6.2) with $x_{i} \in \mathcal{A}\left(n^{1 / k}, n^{\eta}\right)(i=2, \ldots, s)$, where

$$
\mathcal{A}(P, R)=\{x \leq P: p \mid x \Rightarrow p \leq R\},
$$

and $\eta$ is some small constant. This set contains many $P_{r}$-numbers where $r=1 /(\eta k)$ (which we may suppose to be an integer). However, replacing $\mathcal{A}\left(n^{1 / k}, n^{\eta}\right)$ with the set

$$
\mathcal{A}=\left\{p_{1} \ldots p_{r}: 4^{-r} n^{\eta}<p_{i}<n^{\eta}(1 \leq i \leq r)\right\}
$$

puts no serious obstacles in the way of the Hardy-Littlewood work. Using the linear sieve as in this paper, we can solve (6.2) with $x_{j} \in \mathcal{A}(2 \leq j \leq s)$ and some $P_{r^{\prime}}$-number $x_{1}$. We may therefore enunciate the following general principle.

Suppose a bound $G(k) \leq B(k)$ for Waring's problem has been established by the circle method, using the exponential sums

$$
\sum_{x \leq P} e\left(\alpha x^{k}\right), \quad \sum_{x \in \mathcal{A}\left(P, P^{\eta}\right)} e\left(\alpha x^{k}\right) .
$$

Then the methods of this paper are likely to provide the bound $H_{r}(k) \leq B(k)$ for some $r=r(k)$.

In particular, we recall the bounds

$$
G^{+}(4) \leq 12, \quad G(5) \leq 18, \quad G(6) \leq 28, \quad G(k) \leq k(\log k+O(\log \log k)) ;
$$

see $[1,13,15]$ (Vaughan and Wooley have announced further improvements for $G(k)$ when $k \geq 5$ ); here $G^{+}(4)$ is the smallest $s$ such that all large $n \equiv s(\bmod 16)$ are the sum of $s$ biquadrates. In all these cases the principle is readily seen to be valid so that $H_{r}(4) \leq 12, H_{r}(5) \leq 18$ etc., for some $r$. The value of $r$, however, is another matter. The best known bounds for $H_{1}(k)$ (that is, the Waring-Goldbach problem of solving (6.2) in primes) are rather larger; cf. Hua [8].

It may be of interest to note that for large $k$ the exponential sum estimates in Vaughan [13] and Wooley [15] can be modified so as to allow for good control on the sums $\sum_{x \in \mathcal{A}} e\left(\alpha x^{k}\right)$ at individual $\alpha$. This can be used to give a direct circle method proof of the inequality $H_{r}(k) \leq$ $k(\log k+O(\log \log k))$ for some $r=r(k)$, without using sieves. For small values of $k$, however, the sieve is crucial.

We have not yet commented on the quadratic case. In fact, the principle is valid as well for four squares but a substantiation requires refinements of the method; the interested reader is referred to Brüdern and Fouvry [5]. 


\section{References}

[1] J. Brüdern, On Waring's problem for fifth powers and some related topics, Proc. London Math. Soc. (3) 61 (1990), 457-479.

[2] - Sieves, the circle method, and Waring's problem for cubes, Habilitationsschrift, Göttingen 1991; Mathematica Gottingensis 51 (1991).

[3] - , A note on cubic exponential sums, in: Séminaire de Théorie des Nombres, Paris 1990-91, S. David (ed.), Progr. Math. 108, Birkhäuser, Basel, 1992, 23-34.

[4] -, A sieve approach to the Waring-Goldbach problem I: Sums of four cubes, Ann. Sci. Ecole Norm. Sup. Paris, to appear.

[5] J. Brüdern and E. Fouvry, Lagrange's four squares theorem with almost prime variables, J. Reine Angew. Math. 454 (1994), 59-96.

[6] G. Greaves, A weighted sieve of Brun's type, Acta Arith. 40 (1981), 297-332.

[7] L. K. Hua, Some results in additive prime number theory, Quart. J. Math. Oxford 9 (1938), 68-80.

[8] —, Additive Theory of Prime Numbers, Providence, R. I., 1965.

[9] R. C. Vaughan, The Hardy-Littlewood Method, Cambridge University Press, 1981.

[10] - Some remarks on Weyl sums, in: Topics in Classical Number Theory, Colloq. Math. Soc. János Bolyai 34, North-Holland, Amsterdam, 1984.

[11] -, On Waring's problem for cubes, J. Reine Angew. Math. 365 (1986), 121-170.

[12] -, On Waring's problem for cubes II, J. London Math. Soc. (2) 39 (1989), 205-218.

[13] —, A new iterative method in Waring's problem, Acta Math. 162 (1989), 1-71.

[14] G. L. Watson, A proof of the seven cubes theorem, J. London Math. Soc. 26 (1951), $153-156$

[15] T. D. Wooley, Large improvements in Waring's problem, Ann. of Math. 135 (1992), $131-146$.

MATHEMATISCHES INSTITUT A

PFAFFENWALDRING 57

70551 STUTTGART, GERMANY 